\title{
Fuzzy Ontology-Based Querying User' Requests Under Uncertain Environment
}

\author{
Mohamed Nazih Omri, National School of Engineers of Sousse, Sousse University, Tunisia \\ iD https://orcid.org/0000-0001-7803-0179
}

\begin{abstract}
Assistance with the use of technical devices is required as soon as the tasks to be performed become complex. This assistance is also needed as soon as the authors provide assistance to users to find solutions to incidents that occur during the application of unsuitable procedures. The goal of this work is then to provide a knowledge extraction approach that can interpret user requests into valid system requests to respond appropriately to novice user requests. This approach is based on a fuzzy semantic network for the modeling of imprecise and uncertain knowledge and the automatic construction of temporary fuzzy ontology for the identification and interpretation of user requests. The proposed approach has the advantage of being able to integrate the notion of uncertain and imprecise knowledge into the representation of system objects and procedures. The experimental results show the efficiency and the effectiveness of the approach.
\end{abstract}

\section{KEYWORDS}

Fuzzy Ontology, Imprecision, Knowledge Extraction, Uncertainty, User Requests

\section{INTRODUCTION}

The performance of today's conventional help systems is satisfactory in the quality and quantity of information it contains. But their weak point is to identify and interpret the requests of the users expressed in natural language. In fact, in most cases, the user's request does not contain the terms indexing the object and the action to be performed from the point of view of the system. It is a problem of identification and correspondence between the label of the object concerned and that of the goal to be achieved contained in the request and the corresponding entities in the Knowledge Base (KB).

Executing a procedure serves to reach a Goal on an Object. The underlying psychological hypothesis is these Goals are Object properties, and as such, are generators of Object categories. Goals and procedures define the function of Objects and the way to use them. As functional properties of Objects, they enter into the construction of ontology in the same way as structural properties. We define a procedure as a sequence of operations whose execution serves to reach a Goal, and where the elements of the sequence are either primitive actions or subGoals which themselves call for associated procedures. Technical device through procedures can be described on two steps. The first one is the task decomposition as a hierarchy of Goal decomposition into subGoals from the level of the Goal of

This article, originally published under IGI Global's copyright on July 1, 2020 will proceed with publication as an Open Access article starting on February 1, 2021 in the gold Open Access journal, International Journal of Cognitive Informatics and Natural Intelligence (converted to gold Open Access January 1, 2021), and will be distributed under the terms of the Creative Commons Attribution License (http://creativecommons.org/licenses/by/4.0/) which permits unrestricted use, distribution, and production in any medium, provided the author of the original work and original publication source are properly credited. 
the task to primitive actions. The second one consists in (1) drawing up a list of possible Goals and the procedures to reach these Goals and (2) constructing the Ideal Expert Net as a classical ontology.

The goal of this article is to propose a new model based on a Fuzzy Ontology which represents objects and goals of novice users to respond to their fuzzy requests. It is therefore a question of finding through this ontology the objects and procedures of the system(expert) which are the possible equivalents of those of the users. This ontology is considered as an uncertain environment since it establishes possible links, with degrees of possibilities between 0 and 1 , between the objects and procedures of the expert and those of the users. In order to respond appropriately to novice user requests expressed in natural language, we are therefore interested in representing inaccurate and uncertain goals and objects of the novice users in a fuzzy ontology which will then allow us searching and extracting corresponding system's goals and objects.

In the literature, we have not found any work that addresses the problem of identifying queries from novice users by trying to fragment these queries into objects and procedures to identify them separately. And then, using the construction of a fuzzy ontology, we easily identify the object and procedure targeted by the user via the semantic links between the system object / user object and the system procedures / user procedures pairs. This strategy represents the originality of our work compared to the few existing works.

The rest of this paper is organized as follows: Section 2 presents the background. Section 3 provides the needed preliminaries on Fuzzy sets, Linguistic Variables and the Fuzzy Ontologies uses in this contribution. Section 4 is devoted to presenting the architecture of our knowledge extraction approach and its various components. In section 5, we detail the different tests and performance measures performed and we present the results of the experiment. We will conclude with general conclusions and future perspectives for this work.

\section{BACKGROUND}

In this section, we will present the main work done to solve problems similar to our problem. Phan in his work (Phan Cong-Vinh, 2009) proposed series of actions and adaptation relations in his system as a service (SAS); the formal approach proposed consists, in particular, of categorical models and behaviors such that, firstly, AASs, series of actions and adaptation relations will categorically be modeled to provide algebraic frameworks for development of reasoning on their behaviors and, secondly, categorical behaviors of AASs, series of action and adaptation relations will be investigated and developed taking advantage of their categorical models.

In (Akuo Suganuma, Kenji Sugawara, Tetsuo Kinoshita, Fumio Hattori, Norio Shiratori, 2009) the authors proposed in their application system, a hierarchical structure of multi-agents is organized dynamically using heuristics in agents based on the situation of a watched person and watching persons. The system appropriately alters the contents and quality of the live video. The flexible system construction scheme using a multi-agent framework facilitates the symbiosis of RS and DS by bridging the gaps in the care-support service domain.

In (Uchiya, Maemura, Hara, Sugawara, \& Kinoshita, 2009) the authors proposed to raise the efficiency of the agent system design process, they propose an interactive design method of an agent system founded on an agent-repository-based multi-agent framework that emphasizes an important feature of agent system design: the use and reuse of existing agents from an agent repository. They also propose an interactive design environment of agent system (IDEA) and demonstrate its effectiveness.

In (Shanshan, Meng, Yingdong, \& He., 2018) propose the new decision-making methods to the selection of cold chain logistics enterprises under an intuitionists fuzzy environment based on these generalized information aggregation operators, and the proposed method evaluates the scaled prioritized relationships between criteria by priority labels in known and unknown situations. In (Selvachandran, Pal, Alhawari, \& Salleh., 2018), the authors introduce two new operations for the 
interval-valued complex fuzzy set model, and study the fundamental algebraic properties of these new operations. The utility and applicability of the relations of this model is then demonstrated by applying it to an economics problem. Other consider that decision making using fuzzy soft-set and its extensions has become the most significant research area in the age of uncertainty. The evolution of fuzzy soft-sets during the last decade and a half (2001-2015) to analyze the impact of fuzzy soft-sets and its extension in the decision-making paradigm. Based on some selected journals, conferences, and online databases, they classify the decision-making process mainly into ten different categories, which are based on different types of fuzzy soft-sets. They briefly explore each individual category by mentioning the theoretical/algorithmic proposed approaches. Furthermore, all papers are categorized with respect to publication year, published journal, application type, and decision-making criteria. This literature survey provides a platform to the researchers to find out the dimensions of future research works in fuzzy soft-sets by analyzing the present state and potential areas. In (Sooraj \& Tripathy., 2018) authors consider that selection is a challenging task due to the presence of hundreds of varieties of seeds of each kind, some homework is necessary for selecting suitable seeds as new varieties and kinds of seeds are introduced in the market every year having their own strengths and weaknesses. The complexities involved in the characteristics in the form of parameters results in uncertainties and as a result some uncertainty based model or hybrid models of more than is required to model the scenario and come out with a decision. Soft sets have enough of parameterization tools to support and hence is the most suitable one for such a study. The authors proposed then a model called the interval valued fuzzy soft set and propose a decision-making algorithm for the selection of seeds. And they concluded that the use of signed priorities and intervals for the membership of values for entities makes the study more efficient and realistic. In (Chebil, Soualmia, \& Omri., 2018) authors have proposed a new approach titled Conceptual Information Retrieval Model (CIRM). The principle of this contribution is the exploitation of possibilistic networks $(\mathrm{PN})$ and a multi-terminology in order to extract and disambiguate terms and then to retrieve documents. The two measures of possibility and necessity were used to select the relevant concept of an ambiguous term. Thus, the user request and unstructured documents are described throughout a conceptual representation. Concepts were then filtered and ranked. Finally, a possibilistic network was exploited to match documents and requests. Two biomedical terminologies were exploited which are the MeSH thesaurus (Medical Subject Headings) and the SNOMED-CT ontology (Systematized Nomenclature of Medicine of Clinical Terms).

In the following table (see Table 1) we present a summary report of the different approaches studied in order to identify and summarize the advantages and the limits of each of them. The criteria of comparison were chosen as essential characteristics for any interactive system whose objective is to satisfy the fuzzy queries of the users.

\section{FUZZY SETS, LINGUISTIC VARIABLES AND ONTOLOGIES}

Crusty sets are the sets that we have used most of our life. In a crusty set, an element is either a member of the set or not. For example, a jelly bean belongs in the class of food known as candy. Mashed potatoes do not.

\section{Fuzzy Sets}

Fuzzy sets, on the other hand, allow elements to be partially in a set. Each element is given a degree of membership in a set. This membership value can range from 0 (not an element of the set) to 1 (a member of the set). It is clear that if one only allowed the extreme membership values of 0 and 1 , that this would actually be equivalent to crusty sets. A membership function is the relationship between the values of an element and its degree of membership in a set. 
Table 1. Summary report of the different approaches studied

\begin{tabular}{|l|l|l|l|l|}
\hline \multicolumn{1}{|c|}{ Approach } & Used Ontology & $\begin{array}{c}\text { User Request } \\
\text { in Natural } \\
\text { Language }\end{array}$ & $\begin{array}{c}\text { Used Uncertain } \\
\text { Environment }\end{array}$ & $\begin{array}{c}\text { Dynamic } \\
\text { (Enrichissement) }\end{array}$ \\
\hline $\begin{array}{l}\text { Phan aproach } \\
\text { (Phan, 2009) }\end{array}$ & No & No & No & No \\
\hline $\begin{array}{l}\text { Akuo et al. approach } \\
\text { (Akuo et al., 2009) }\end{array}$ & No & Yes & No & Yes \\
\hline $\begin{array}{l}\text { Takahiro approach } \\
\text { (Takahiro et al., 2009) }\end{array}$ & No & No & Yes & No \\
\hline $\begin{array}{l}\text { Shanshan et al. approach } \\
\text { (Shanshan, et al., 2018) }\end{array}$ & No & No & Yes & No \\
\hline $\begin{array}{l}\text { Ganeshsree et al. approach. } \\
\text { (Ganeshsree et al., 2018) }\end{array}$ & No & No & Yes & Yes \\
\hline $\begin{array}{l}\text { Sooraj and Tripathy approach } \\
\text { (Sooraj and Tripathy, 2018) }\end{array}$ & No & No & Yes & No \\
\hline $\begin{array}{l}\text { Chebil et al. approach } \\
\text { (Chebil et al., 2018) }\end{array}$ & Mesh & Yes & Yes & No \\
\hline
\end{tabular}

\section{Linguistic Variables: From Crusty Sets to Fuzzy Sets}

Fuzzy logic is an extension of classical logic where truth propositions can take any number between 0 and 1 to model imprecise and partially true statements. Classical logic describes a binary truth proposition, where a proposition is true if an element $\mathrm{e}$ is a member of the set $\mathrm{A}$ of interest (i.e. $\mathrm{e} \in$ $A=1$ ), and a proposition is false if an element $\mathrm{e}$ is not part of set $\mathrm{A}$ (i.e. $\mathrm{e} \in \mathrm{A}=0$ ). These binary sets are said to be crusty, and they are represented by a step membership function $\mu_{\mathrm{A}}$ which maps the element e to the set A if A is completely representative of e. In Figure 1, the membership function assigns heights below six feet to the set not tall and assigns heights above six feet to the set tall. A five feet ten inches person is classified as not tall and a six feet two inches person is classified as tall. Formally, a crusty set or classical set A is defined as follows.

Figure 1. Crusty membership function describing human height

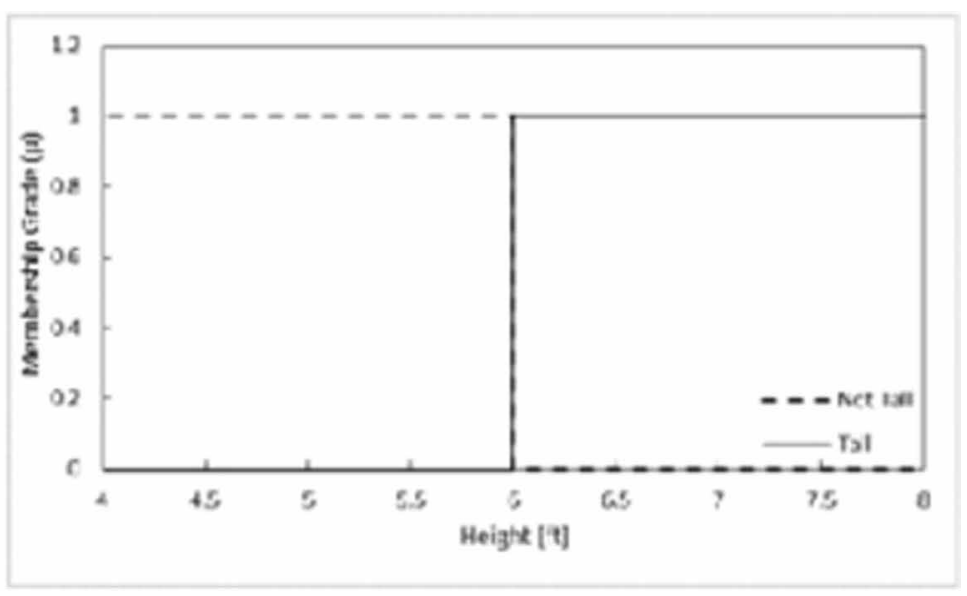


Definition 1: A crusty set is comprise of a domain $X$ of the real numbers (also called the universe of discourse of $A)$ together with a membership function $\mu_{A}(x)$, where $\mu_{A}(x)$ is given by the indicator function in Equation 1:

$\mu_{A}(x)= \begin{cases}1, & \text { if } x \in A \\ 0, & \text { else }\end{cases}$

Fuzzy set theory expands this concept to allow the degree of membership of an element to a set to lie between 0 and 1 (i.e the membership function is not restricted to an indicator function). Thus, a classical set is a special case of a fuzzy set. Two classes of fuzzy sets existfirst type fuzzy sets and second type fuzzy sets-which differ by their ability to handle linguistic uncertainties. A first type fuzzy set is defined by a single deterministic, or crusty, membership function. Once it is defined, all linguistic uncertainty about the membership function disappears. In second type fuzzy sets membership functions are themselves uncertain, or fuzzy, and can take any value within their defined bounds.

\section{First Type of Fuzzy Sets}

The first type of fuzzy sets are the most widely used, and have been successfully applied to many real world problems. They model linguistic uncertainty through the use of a crusty membership function, which determines the similarity between a numerical variable (i.e., element e) and a linguistic variable. Formally, a fuzzy set is defined by Mendel et al. (2014) as follows.

Definition 2: A fuzzy set A is comprised of a domain $X$ of the real numbers (also called the universe of discourse of $A$ ) together with a membership function $\mu_{A}: X \rightarrow(0,1)$. For each $x \in X$, the value of $\mu_{A}(x)$ is the degree of membership, or membership grade, of $x$ in $A$. If $\mu_{A}(x)=1$ or $\mu_{A}(x)=0 \forall$ $\mathrm{x} \in \mathrm{X}$, then the fuzzy set is said to be a crusty set.

A First type of fuzzy set A is described as in Equation 2, where the integral operator denotes the collection of all values $x$ in the universe of discourse $X$, with degree of membership $\mu_{A}(x)$. Additionally, the slash does not denote division. It associates the value $\mathrm{x}$ to a degree of membership in set $\mathrm{A}$ :

$\mu_{A}(x)=\int_{x i n A} \mu_{A}(x) / x$

The non-binary degree of membership of a numerical variable $\mathrm{x}$ to the set $\mathrm{A}$ better captures the nuances of real life and the imprecisions associated with human reasoning. The sharp boundary used by the crusty membership function to categorize human height given in Figure 1 is unnatural, as there is no sharp boundary describing a change from tall to not tall. Instead, humans tend to think of a gradual change from the height of a tall person to the height of a person who isn't. The fuzzy membership function shown in Figure 2 captures this imprecise boundary and gradual change. The five feet ten inches person and the six feet two inches person are both about half tall and half not tall, with the first being slightly more not tall and the second being slightly more tall.

\section{Second Type of Fuzzy Sets}

The second type of fuzzy sets expand their first type counterpart to incorporate uncertainty in their membership function definition. A major criticism of the first sets is that they are themselves nonfuzzy, which limits their ability to handle uncertainty in their membership function definition. Words mean different things to different people, and are also context dependent. This concept cannot be 
Figure 2. Exemple of fuzzy membership function describing human height

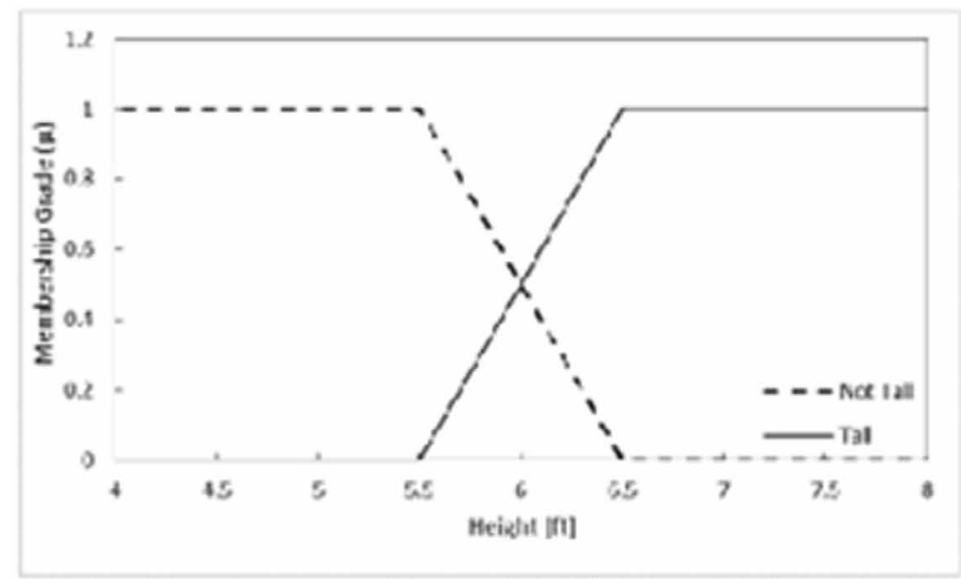

adequately captured by first type sets. Figure 2 shows a precise definition of the membership functions for tall (i.e., there is no uncertainty associated with its end points); however, a person's definition of the concept tall tends to be vague and to depend on several factors, like nationality and age. Using a first type set with an exact membership function seems counter-intuitive when describing a fuzzy quantity which is by definition imprecise. The second type of sets, or fuzzy-fuzzy sets, provide a more accurate model of linguistic uncertainty by using a fuzzy definition of the primary membership function (Figure 3). They are defined by their primary membership $\mu_{\mathrm{A}}$, which is plotted into the

Figure 3. Exemple of fuzzy membership function describing human height

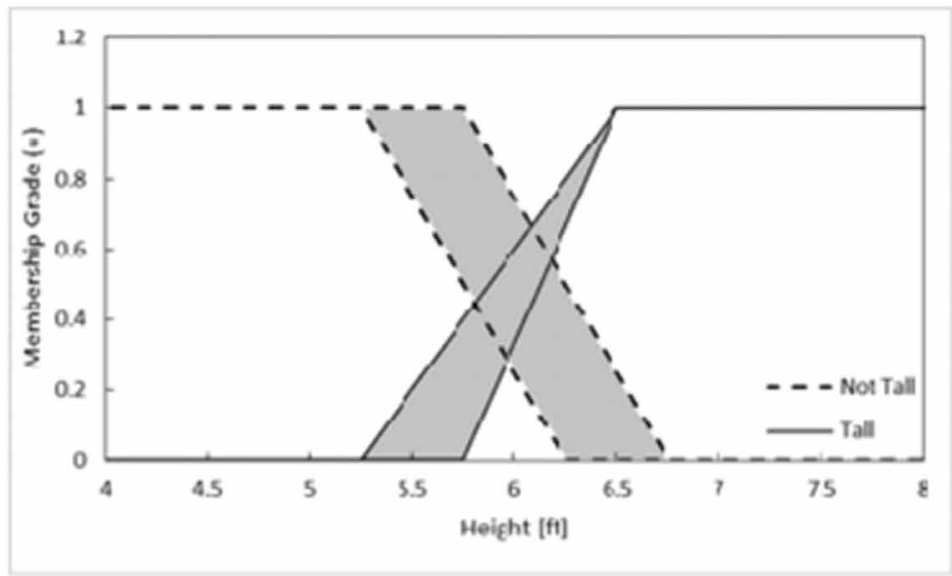

plane of the paper, and their secondary membership $u$, which is plotted on the vertical axis. The shaded area is referred to as the footprint of uncertainty (FOU) of the primary membership function, and is bounded below by the lower membership function (LMF) and bounded above by the upper membership function (UMF). The even shading implies that all primary memberships between the LMF and UMF are equally likely and thus uniformly weighted. Such sets are denoted interval the second type of fuzzy sets, and make up virtually all applications of the second type of fuzzy sets. 
Fuzzy sets with non-uniform secondary membership functions are called general of second type of fuzzy sets, but are still in infancy. If all uncertainty about membership function definition disappears, the secondary membership u reduces to $\mu_{\mathrm{A}}$ and a second type of set reduces to a first type set, much like a random variable reduces to the mean when the variance goes to zero.

One can also think of a second type of fuzzy set as a collection of an infinite number of embedded first type fuzzy sets contained within the shaded area and bounded between the LMF and UMF. For interval second type of sets, each of these embedded functions is equally weighted.

A second type of fuzzy set A can be described as in Equation 3, where the first integral operator denotes the collection of all values $\mathrm{x}$ in the universe of discourse $\mathrm{X}$, and the second integral denotes the collection of all secondary membership $\mathrm{u} \in \mathrm{U} \subseteq(0,1)$ of its embedded first type sets:

$$
\begin{aligned}
& \mu_{A}(x)=\int_{x \in X} \int_{u \in U_{x}} \mu_{A}(x, u) /(x, u) \\
& \mu_{A}(x)=\int_{x \in X} \int_{u i n(0,1)} 1 /(x, u)=1 / \operatorname{FOU}(A)
\end{aligned}
$$

\section{Fuzzy Ontologies for Extracting Relevant Information}

We represent here networks used in the search for information that allow us to represent associative relations between the different terms. Thesauri allow for example to extend a q request to synonym terms. One can either consider binary relations between terms, or consider fuzzy relationships as Radecki (Radecki, 1976) proposed. In the latter case, we speak of fuzzy ontologies. Lucarella and Morara (Lucarella \& Morara, 1991) present an example of an information search system based on networks of fuzzy concepts. They consider a network of concepts in which the links are oriented and valued and in which also nodes representing the documents appear (Figure 4). Link valuation represents the degree to which the concept or document being pointed to relevant to the origin node.

The concepts are usually words (the key words chosen for the representation of documents) and the automatic construction of ontologies most often consists of measuring the co-occurrence of words in the different documents. So when two words appear in the same documents, they are perfectly linked.

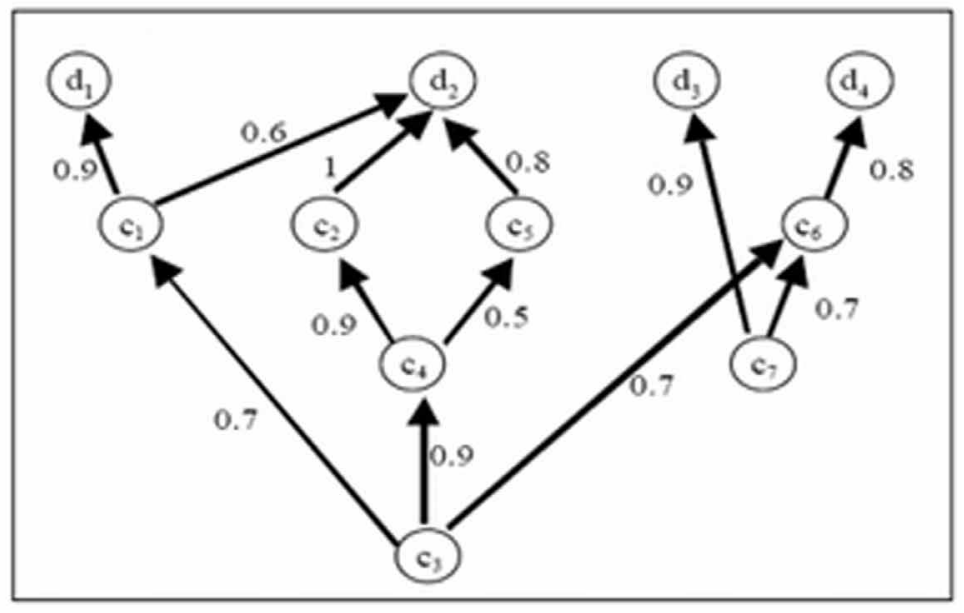




\section{PROPOSED APPROACH}

The General architecture of the proposed approach is given by Figure 5. The example of a technical system that we consider is an airplane piloting system whose objects are such as airplane, joystick, throttle, and the procedures are such as take off, turn left, or lowering.

\section{Figure 5. General architecture of the proposed approach}

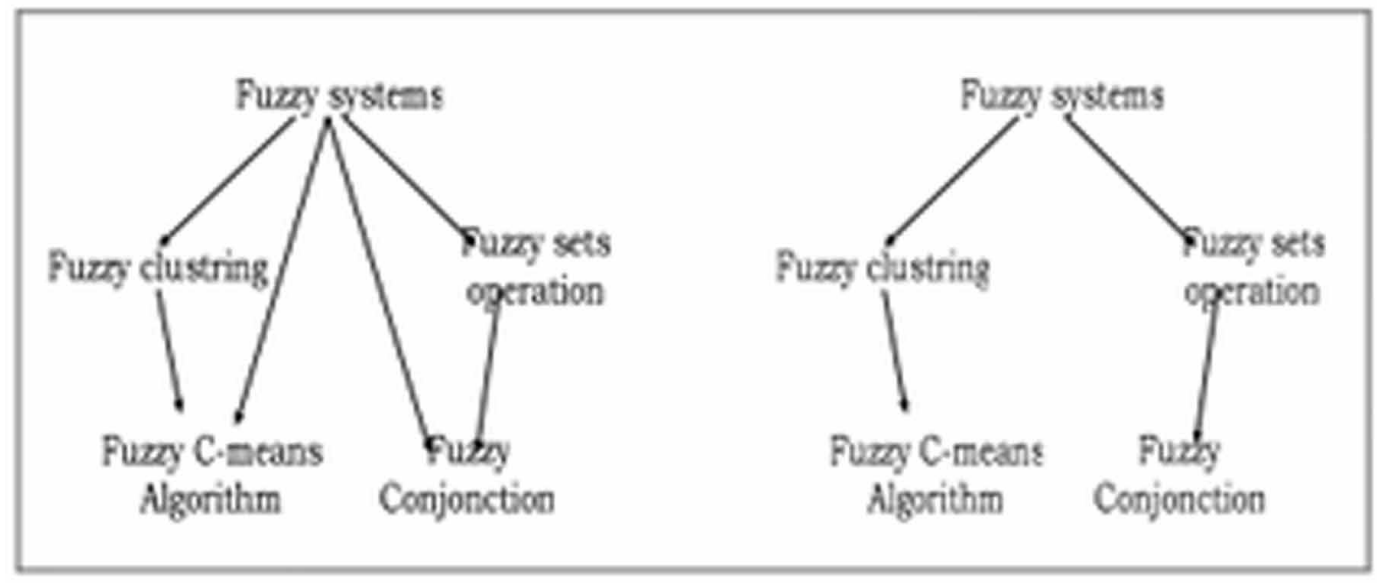

The model we proposed offers the user the ability to enter a user request that can be in the following form: "How to make take off the craft?" The extraction model that we propose allows to build a valid system request such as "how to take off the plane?" equivalent to the initial request user.

The analysis of the user request is based on the search and retrieval of relevant knowledge from the fuzzy semantic network that forms the core of the KB. It also uses fuzzy ontology construction to estimate the relevance of user requests against equivalent system requests (Figure 6).

\section{Structures of System Objects and Procedures}

\section{Structures of the System Objects}

Let $\mathrm{O}_{\mathrm{S}}$ be a system object and $\mathrm{O}_{\mathrm{U}}$ be the user object. We associate with each system object a list users denoted by $\mathrm{Z}_{\mathrm{Os}}$ and extracted from novice user requests expressed in natural language. For each object in the list, we associated a degree dij of belonging $\mu_{\mathrm{Ou}_{i} / O_{s}}$ which reflects the degree to which we believe that the semantic meaning of $\mathrm{O}_{\mathrm{ui}}$ is the user object $\mathrm{O}_{\mathrm{S}}$ is near to the semantic meaning of the targeted system object $\mathrm{O}_{\mathrm{S}}$. The list users denoted by $\mathrm{Z}_{\mathrm{Os}}$ is given by the next equation (Equation 5):

$$
Z_{O s}=\left\{\left(O_{U_{1}}, d_{11}\right),\left(O_{U_{2}}, d_{12}\right), \ldots,\left(O_{U_{n}}, d_{1 n}\right)\right\}
$$

\section{Example:}

$$
Z_{\text {air-plain }}=\{(\text { machine }, 0.5),(\text { device }, 0.6), \ldots,(\text { engine }, 0.2)\}
$$




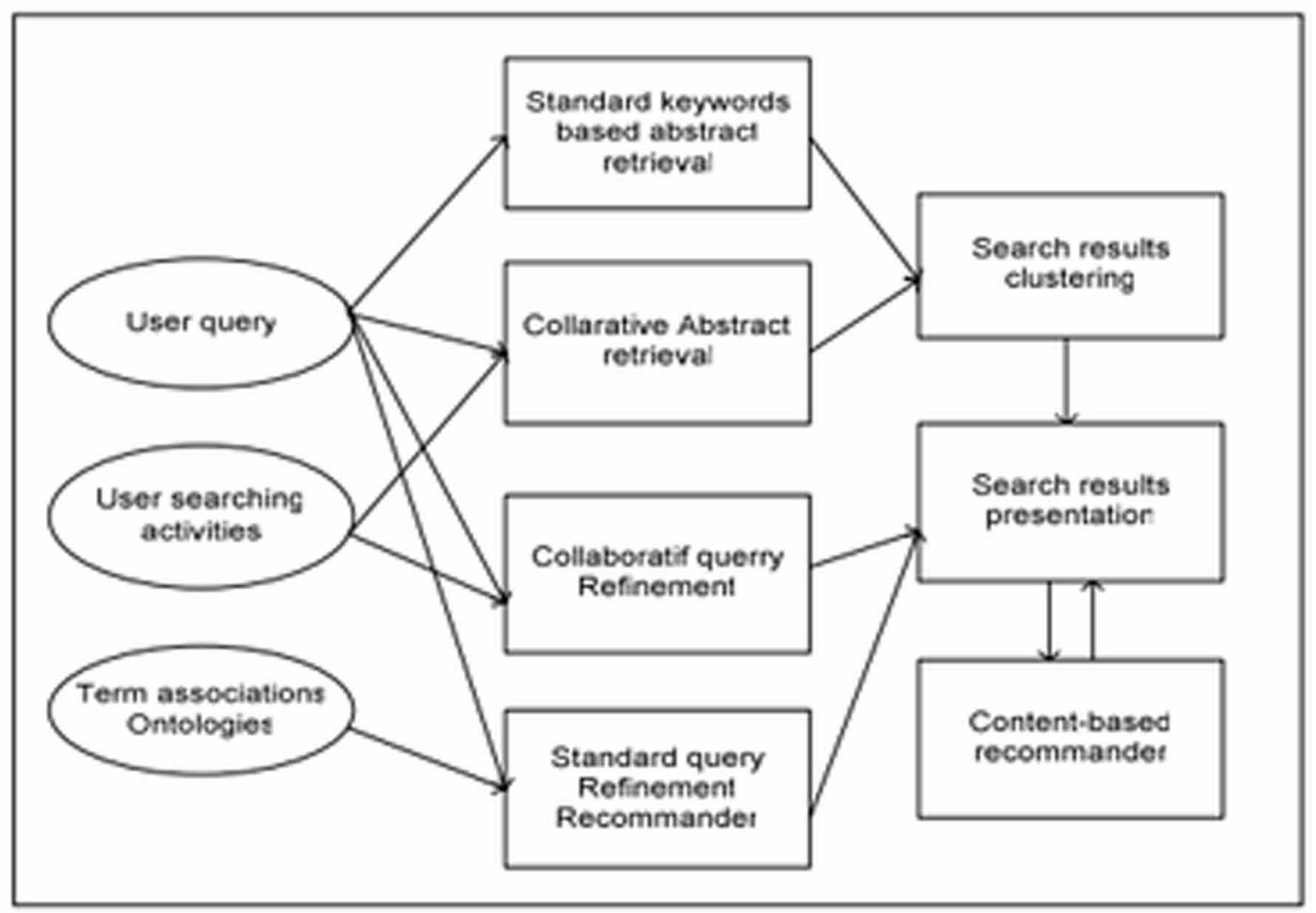

\section{Structure of the System Procedures}

As with system objects, we have associated with each system procedure belonging to a system class a "user zone" consisting of a list of procedures used by users to designate the system procedure and the degrees with which we believe these procedures have been performed. A semantic meaning close to the meaning of the intended system procedure.

Example:

$$
Z_{\text {remove }}=\{(\text { start }, 0.3),(\text { move }, 0.6), \ldots,(\text { leave }- \text { the }- \text { ground }, 0.8)\}
$$

To increase the performance of search engines on the Internet, new methods are implemented that not only look for terms present in the request, but they also allow to find other terms that are related to those that are sought. These methods use what is called "fuzzy ontologies".

\section{Ontology Construction Process}

The principle of our approach algorithm for the automatic construction of fuzzy ontologies is based on two principal steps. Let us given some definitions that will allow us the construction of the ontology. For this reason, let $\mu_{\mathrm{Occ}}\left(\mathrm{t}_{\mathrm{ij}}, \mathrm{a}_{\mathrm{i}}\right)$ be the function which characterizes the occurrences of term $t_{i j}$ in article $\mathrm{a}_{\mathrm{i}}$.

Definition 3: Let $C=\left\{a_{1}, a_{2}, \ldots, a_{n}\right\}$ be a collection of articles $a_{i}$ and each article $a_{i}=\left(t_{1}, t_{2}, \ldots, t_{m}\right)$ is represented by a set of terms $t_{j}$ and NT $\left(t_{i}, t_{j}\right)$ be the function which expresses narrow-than $t_{j}$. We defined the belonging degree $\mu_{\mathrm{NT}}\left(\mathrm{t}_{\mathrm{i}}, \mathrm{t}_{\mathrm{j}}\right)$ by the next equation (Equation 6): 
$\mu_{N T}\left(t_{i}, t_{j}\right)=\frac{\sum_{a \in C} \min }{\sum_{a \in C} \mu O c c}$

$\mu_{\mathrm{NT}}\left(\mathrm{t}_{\mathrm{i}}, \mathrm{t}_{\mathrm{j}}\right) \approx 1$ when $\mathrm{t}_{\mathrm{i}}$ and $\mathrm{t}_{\mathrm{j}}$ are present together in the articles or when the occurrences of $\mathrm{t}_{\mathrm{i}}$ are much smaller than those of $t_{\mathrm{j}}$.

Definition 4: Let $\mu_{\mathrm{BT}}\left(\mathrm{t}_{\mathrm{i}}, \mathrm{t}_{\mathrm{j}}\right)$ be the broader-than function that denotes $\mathrm{t}_{\mathrm{i}}$ is wider than $\mathrm{t}_{\mathrm{j}}$. Because broader is the opposite of narrower, this measure is given by the next equation (Equation 7):

$\mu_{B T}\left(t_{i}, t_{j}\right)=\mu_{N T}\left(t_{j}, t_{i}\right)$

Definition 5: The third relationship, called Related-Term (RT) is defined by the next equation (Equation 8):

$\mu_{R T}\left(t_{i}, t_{j}\right)=\frac{\sum_{a \in C} \min (}{\sum_{a \in C} \max (}$

\section{First Step of Ontology Construction Process}

The first is to build a complete ontology from NT relationships. For each pair of terms $\left(t_{i}\right.$, $\left.t_{j}\right)$, we compute the membership degrees of two NT relations, $\forall \mathrm{t}_{\mathrm{i}}, \mathrm{t}_{\mathrm{j}} \in \mathrm{a}_{\mathrm{i}} / \mathrm{t}_{\mathrm{i}} \neq \mathrm{t}_{\mathrm{j}}$, calculate $\mu_{\mathrm{NT}}\left(\mathrm{t}_{\mathrm{i}}, \mathrm{t}_{\mathrm{j}}\right)$ and $\mu_{\mathrm{NT}}\left(\mathrm{t}_{\mathrm{j}}, \mathrm{t}_{\mathrm{i}}\right)$.

We keep the NT relation which has the highest degree, $\max \left(\mu_{N T}\left(t_{i}, t_{j}\right), \mu_{N T}\left(t_{j}, t_{i}\right)\right)$ and we eliminate the other NT relationship. In this way, the NT redundant relationships are eliminated and the stored information is halved.

When the relationships between the terms are not strong, the values of their NT relations have low degrees, and therefore cannot be incorporated into the ontology. One fixes a certain limit $\alpha$, then one applies ( $\alpha$-cut) which consists of removing all the NT relations which are below this limit. If an NT relation has a degree equal to zero $\mu_{\mathrm{NT}}\left(\mathrm{t}_{\mathrm{i}}, \mathrm{t}_{\mathrm{j}}\right)=0$, then the two terms are unrelated.

\section{Second Step of Ontology Construction Process}

This step consists of reducing the ontology by removing all NT relations not necessary. Figure 3 shows two ontologies, one with excessive relationships, and the other normal. From this it can be noticed that two terms can have a relation either directly or via other terms "indirect paths". We begin by finding all the indirect paths between the two terms $t_{i}$ and $t_{j}$.

Definition 6: Let $P=\left\{N T\left(t_{i}, t_{m 1}\right), N T\left(t_{m 1}, t_{m 2}\right), \ldots, N T\left(t_{m p}, t_{j}\right)\right\}$ be one of these paths. NT(P) is assumed to be an NT alternative relation for $\left(t_{i}, t_{j}\right)$. The degree of adhesion of NT(P), which is defined by the next equation (Equation 9) as follows:

$$
\mu_{N T}(P)=\min \left\{N T\left(t_{i}, t_{m 1}\right), N T\left(t_{m 1}, t_{m 2}\right), \ldots, N T\left(t_{m p}, t_{j}\right)\right\}
$$

\section{Identification Algorithm of User Objects and Procedures}

The identification process consists of returning the most appropriate system object to the user object expressed in natural language. The idea we propose is not only to exploit the degree of belonging of 
the user object of the request to the different system objects, but to build a S consisting of objects fuzzy ontology to connect this object with other domain objects. The goal is to find the most relevant path for linking the user object to the target system object.

\section{Different Steps of the Algorithm}

Let $\mathrm{o}_{\mathrm{Ux}}$ be the user object contained in the request, $\mathrm{o}_{\mathrm{Si}}$ be the system object, $\mathrm{o}_{\mathrm{Ui}}$ be the user object and $\mathrm{Z}_{\mathrm{OSi}}$ be the user area of the system object $\mathrm{o}_{\mathrm{S}}$.

The first step of the algorithm is to extract the user objects that will be added to the ontology. These objects must check the condition $\mu_{\text {OUi/OSk }}>\mu_{\text {OUx/OSk }}$ and $o_{U x}$ et $o_{U i} \in Z_{\text {OSk }}$. This condition is necessary to select only the user objects that have a more meaning narrow than the initial object of the user. The extracted objects are added to the list $\mathrm{L}=\left\{\mathrm{o}_{\mathrm{Ux}}, \mathrm{o}_{\mathrm{U} 1}, \mathrm{o}_{\mathrm{U} 2}, \ldots, \mathrm{o}_{\mathrm{Um}}\right\}$.

The second step of the algorithm consists of two different phases. In the first phase, we will build the ontology from the list L of user objects using the NT relationship (narrower than) between the two objects $\mathrm{o}_{\mathrm{Ui}}$ and $\mathrm{o}_{\mathrm{Uj}}$. The narrower than degree between two terms $\mathrm{t}_{\mathrm{i}}$ and $\mathrm{t}_{\mathrm{j}}$ noted $\mu_{N T}\left(o_{U i}, o_{U i}\right)$ is defined by the following equation (Equation 10):

$$
\mu_{N T}\left(t_{i}, t_{j}\right)=\frac{\sum_{k} \min }{\sum_{k} \mu O U i, O S k}
$$

With $\mu_{\mathrm{NT}}\left(\mathrm{o}_{\mathrm{Ui}}, \mathrm{o}_{\mathrm{Uj}}\right)$ is the relevance degree which means that the object $\mathrm{o}_{\mathrm{Ui}}$ is more relevant than $\mathrm{o}_{\mathrm{Uj}}$. In this step, the relevant system objects are added to the ontology to get the final fuzzy ontology.

In third step we proceed to measure the relevance degree $d_{i}$ of the different system objects with respect to the user object to build the list of equivalent system objects sorted in order of decreasing relevance.

The input parameter of our algorithm is therefore a user object (or a procedure) and the output is a set $\mathrm{m}$ of objects (or procedures) systems sorted by degree of relevance $\mathrm{d}_{\mathrm{i}}$ decreasing.

\section{Building of the Equivalent System Requests}

Algorithm: Construction of the ontology

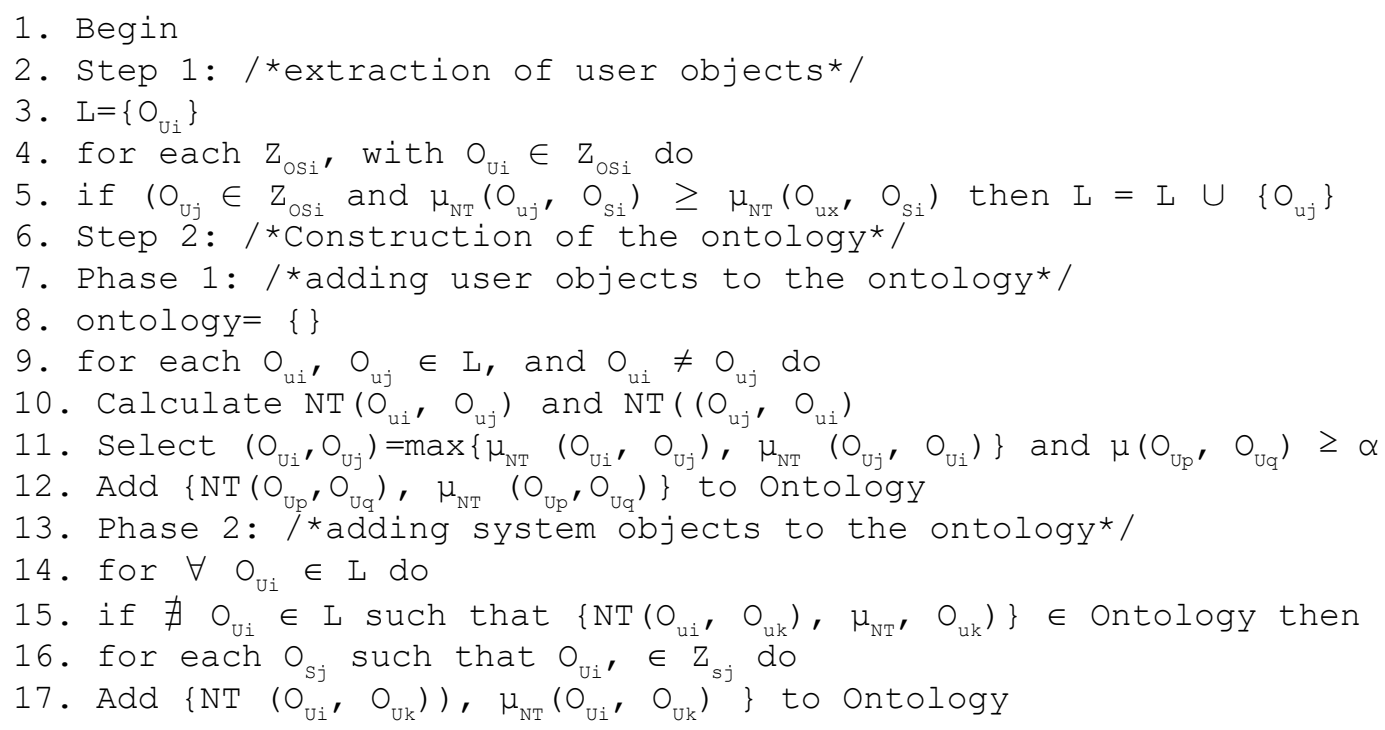


18. if $\exists O_{S j}$ such that $O_{U i} \in Z_{\text {osj }}$ and $\nexists S_{o_{u_{i}}} \rightarrow_{o_{s_{j}}}$ then

19. Add $\left\{\mathrm{NT}\left(\mathrm{O}_{\mathrm{Ui}}, \mathrm{O}_{\mathrm{Sj}}\right), \mu_{\mathrm{NT}}\left(\mathrm{O}_{\mathrm{Ui}}, \mathrm{O}_{\mathrm{Sj}}\right)\right\}$ to Ontology

20. Step 3: / ${ }^{*}$ Computation of the relevance degrees*/

21. Let $S_{o_{u_{i}}} \rightarrow o_{s_{i}}$ be the maximum path that binds $o_{U_{x}}$ to $o_{S_{i}}$

22. $\operatorname{Pert}\left(O_{U x}, O_{S i}\right)=\frac{\sum_{i=1, n}}{n}$

23. end.

$\nexists S_{o_{u_{i}}} \rightarrow_{o_{s_{i}}}$ : means that there is not a path that binds $O_{u i}$ to $O_{s j}$

$\mathrm{n}$ : is the number of lies in $S_{O x} \rightarrow_{O_{S i}}$ and $\mu_{\mathrm{NT}}$ is the value attributes

to the link.

The construction of the equivalent system requests proposed to the user is based on the identification algorithm (see Algorithm 1). After having identified the object and the user procedure, the algorithm for constructing equivalent system requests proposes to the user, based on the set of objects and procedures identified, a set of possible requests which are associated degrees of relevance.

Example:

Let's take the example where the user request is: How to start the craft? We seek to identify the system object that corresponds to the user object "engin", for this reason we will use the proposed identification algorithm.

The different user areas of the objects airplane, motor, and steering wheel are defined as follows:

$$
\begin{aligned}
& Z_{\text {air-plain }}=\{(\text { engin }, 0.5),(\text { apparatus }, 0.6), \ldots,(\text { machine }, 0.2)\} \\
& Z_{\text {motor }}=\{(\text { Turbine }, 0.4),(\text { engin }, 0.5), \ldots,(\text { machine }, 0.6)\} \\
& Z_{\text {steeringwheel }}=\{(\text { device }, 0.6),(\text { joystick }, 0.4), \ldots,(\text { machine }, 0.2)\}
\end{aligned}
$$

\section{Step 1: Extract the User Objects}

Initially we have $\mathrm{L}=\left\{\mathrm{o}_{\mathrm{ui}}\right\}=\{$ machine $\}$. Since engine belongs to the user zones $\mathrm{Z}_{\text {aircraft }}$ and $\mathrm{Z}_{\text {motor? }}$, we will add the user objects, that have a membership degree greater-than or equal to engin, to $L=\{$ engin, device, machine $\}$.

Step 2: Build the fuzzy ontology

Phase 1: Adding User Objects to the Ontology

In this phase we will build the fuzzy ontology from the constructed list $\mathrm{L}$ and we will use the equation (Equation 2):

$$
\begin{aligned}
& \mu_{N T}(\text { engin, device })=\frac{\min (0.5,0.6)+\min (0.5,0)}{0.5+0.5}=0.5 \\
& \mu_{N T}(\text { device,engin })=\frac{\min (0.6,0.5)+\min (0,0.5)}{0.6}=0.83
\end{aligned}
$$


$\mu_{N T}($ engin, machine $)=\frac{\min (0.5,0.2)+\min (0.5,0.2)}{0.5+0.5}=0.8$

$\mu_{N T}($ machine, engin $)=\frac{\min (0.2,0.5)+\min (0.6,0.5)}{0.2+0.6}=0.87$

$\mu_{N T}($ device, machine $)=\frac{\min (0.6,0.2)+\min (0,0.6)}{0.6}=0.33$

$\mu_{N T}($ machine, device $)=\frac{\min (0.2,0.6)+\min (0.6,0)}{0.2+0.6}=0.25$

Phase 2: Adding system objects to the ontology.

We notice that the user objects device and machine do not have NT relations to other user objects of L. They can therefore be linked to the airplane and engine system objects since they appear in their zones. We obtain the final ontology (see Figure 7).

Figure 7. Final fuzzy ontology obtained from the engin user object

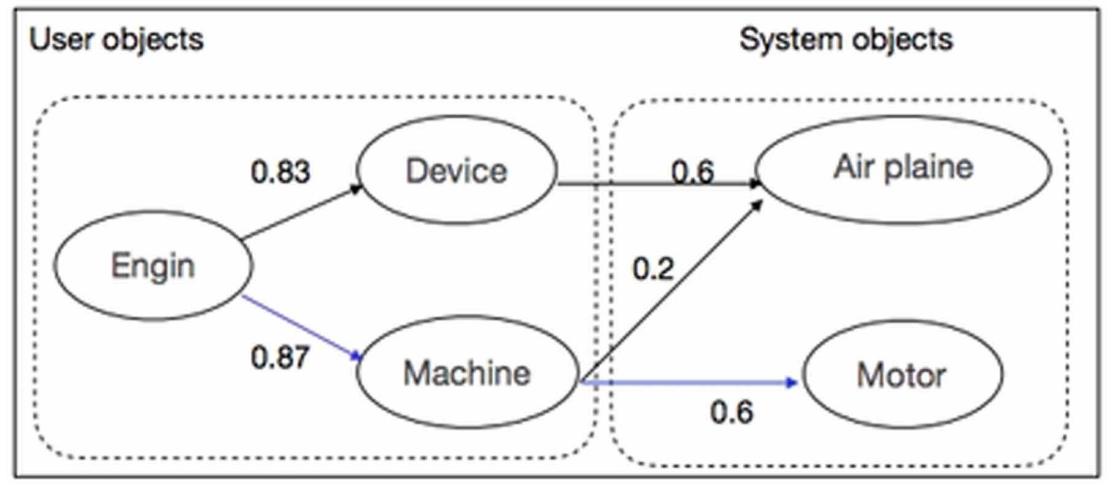

Step 3: Counting of the relevance degrees.

The relevance of a system object $\mathrm{O}_{\mathrm{Si}}$ with regard to a user object $\mathrm{O}_{\mathrm{Ux}}$ is calculated as follows:

$$
\operatorname{Pert}\left(S_{\text {engin,airplaine }}\right)=\frac{0.83+0.6}{2}=0.715 \text { and } \operatorname{Pert}\left(S_{\text {engin,motor }}\right)=\frac{0.87+0.6}{2}=0.73
$$

\section{EXPERIMENTAL STUDY AND RESULTS ANALYSIS}

We can easily notice that these works of literature try to help the users to satisfy their objects. But none of them use a fuzzy ontology to measure the semantic links between user and system objects and procedures. For this reason, we have not been able to implement the literature approaches to compare them to ours and we have, instead, propose to use standard metrics to measure and evaluate 
the performance of our approach. We consider a number of relevant and irrelevant requests that were rendered during the analysis of a user requests. We used three standard relevance coefficients which are recall, precision and f-measure coefficients.

Suppose a system has returned 10 objects (or procedures) in response to a request. Suppose also that a judge has been shown and that he considers that only 5 objects (or procedures) are relevant. We will say that the system has an accuracy of $50 \%$. This would work perfectly without a small problem. This is due to the fact that only 5 objects (or procedures) are relevant throughout the collection. Finding the 5 objects (or procedures) would be a perfect result, and should not be seen as just $50 \%$ success. Therefore, one needs to define another measure as the ratio of relevant objects (or procedures) found on relevant objects (or procedures) throughout the collection.

For this measure, our example will be $5 / 5$, so $100 \%$. This measure is commonly called a reminder, whereas the first one measures the ratio of objects (or procedures) found relevant to the total of objects (or procedures) found, is called precision.

Precision and recall measurements are obtained by partitioning all the objects (or procedures), restored by the system into two categories: the relevant objects (or procedures) and the irrelevant objects (or procedures).

The recall coefficient measures the ability of the system to retrieve all relevant system requests responding to the initial request of the novice user. It is given by the ratio of the relevant found system requests to the set of system requests in the $\mathrm{KB}$.

Definition 7: Let $\mathrm{R}$ be the number of system requests in the $\mathrm{KB}$ and $\mathrm{R}_{+}$be the number of relevant system requests returned from the KB during the analysis of a user request $\left(\mathrm{q}_{\mathrm{i}}\right)$. We defined the recall coefficient by the following equation (Equation 11):

$$
\operatorname{Recall}\left(q_{i}\right)=\frac{R_{+}}{R}
$$

Precision measures the ability of the system to reject all irrelevant system requests to the user request expressed in natural language. It is given by the ratio between the set of relevant selected requests and the set of selected system requests.

Definition 8: Let $R_{+}$be the number of relevant system requests returned from the $\mathrm{KB}$ to the during the analysis of a user request $q_{i}$ and $M$ be the number of system requests returned from the $K B$ that are relevant to the user request $q_{i}$. We define the precision degree of the request $q_{i}$ by the following equation (Equation 12):

$$
\operatorname{Precision}\left(q_{i}\right)=\frac{R_{+}}{M}
$$

Definition 9: We define the F-measure coefficient of the request $\mathrm{q}_{\mathrm{i}}$ as a measure that combines precision and recall measures which is their harmonic mean, by the following equation (Equation 13):

$$
F-\text { measure }\left(q_{i}\right)=2 * \frac{\text { Precision } * \text { Recall }}{\text { Precision }+ \text { Recall }}
$$


To evaluate the performance of the model, we have developed our own KB for the flight control system from the semantic network structure inspired by flight simulation software (flight simulator, 2004) and validated by an experienced pilot of the air force (Table 2).

Table 2. Characteristics of the test KB

\begin{tabular}{|l|l|}
\hline \multicolumn{1}{|c|}{ Data } & \multicolumn{1}{c|}{ Number } \\
\hline System objects & 26 \\
\hline System procedures & 38 \\
\hline System attributes & 10 \\
\hline Possibilistic system requests & 38 \\
\hline
\end{tabular}

We have prepared a set of user requests that we will apply to our approach (Table 3).

To study the behavior of the proposed approach, following is the insertion of new objects by learning, we tested user requests on three sets (see Table 4).

We have measured the average accuracy of our extraction model for the different sets defined in Table 4 . We have noticed that our model offers a good precision $(>0.4)$ when the number of proposed

Table 3. Characteristics of user requests

\begin{tabular}{|l|l|}
\hline \multicolumn{1}{|c|}{ Data } & \multicolumn{1}{c|}{ Number } \\
\hline User requests & 30 \\
\hline Requests with known objects and unknown procedure & 8 \\
\hline Requests with unknown objects and known procedures & 8 \\
\hline Requests with unknown objects and procedures & 14 \\
\hline
\end{tabular}

Table 4. Characteristics of databases used sets of user requests

\begin{tabular}{|l|l|l|}
\hline \multicolumn{1}{|c|}{ Data Base } & \multicolumn{1}{|c|}{ Number of User Objects } & \multicolumn{1}{c|}{ Number of User Procedures } \\
\hline First data set: Set 1 & 26 & 36 \\
\hline Second data set: Set 2 & 52 & 72 \\
\hline Third data set: Set 3 & 78 & 108 \\
\hline
\end{tabular}

requests is low, it is that is, the percentage of requests retrieved is in the range $(0.07,0.3)$. We have also been able to notice that when the number of requests extracted is quite high, the accuracy of the proposed approach decreases while remaining higher than 0.1 and thus there will always be relevant requests among the requests proposed to the user (see Figure 8).

We also observed an improvement in the proposed approach accuracy for the curves of data set 1 and data set 2 , especially when the number of requests selected by our extraction model is high. We explained this phenomenon by the fact that the enrichment of the KB allowed our model to perfect the extraction process. 
Figure 8. Measurement of the precision coefficient

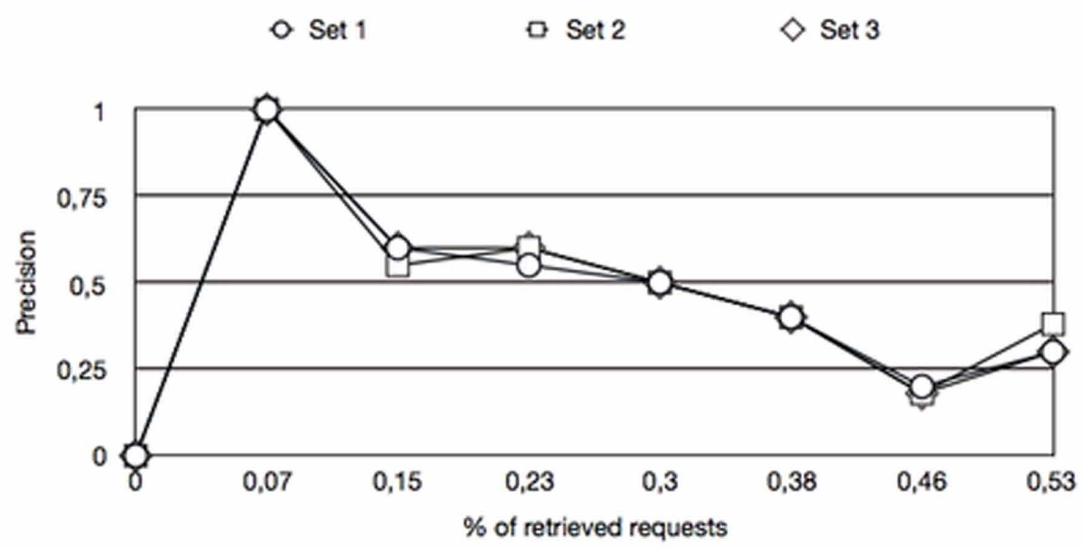

We measured the recall rate of our proposed model for the different sets of the KB. Figure 9 shows that the recall rate varies in the range $(0.45,1)$. This shows us that the majority of the system requests equivalent to the initial request of the novice user were selected and extracted by our extraction model. Especially for the curve of the set 2 and the curve of the set 3, we notice that the recall rate is greater than 0.6 .

Figure 9. Measurement of the recall coefficient

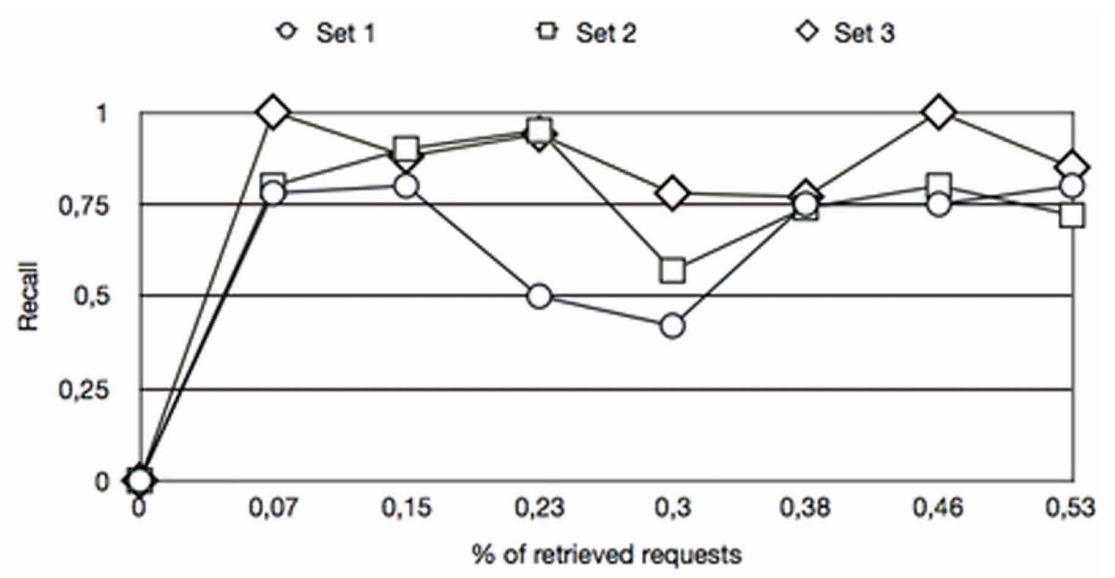

We also measured the F-measure rate to prove the efficiency of our proposed model for the different sets of the KB. Figure 10 shows that the F-measure rate varies in the range $(0.32,1)$. This values shows that the majority of the system requests equivalent to the initial request of the novice user were selected and extracted by our extraction model. Especially for the three curves, we notice that the recall rate is greater than 0.6. 


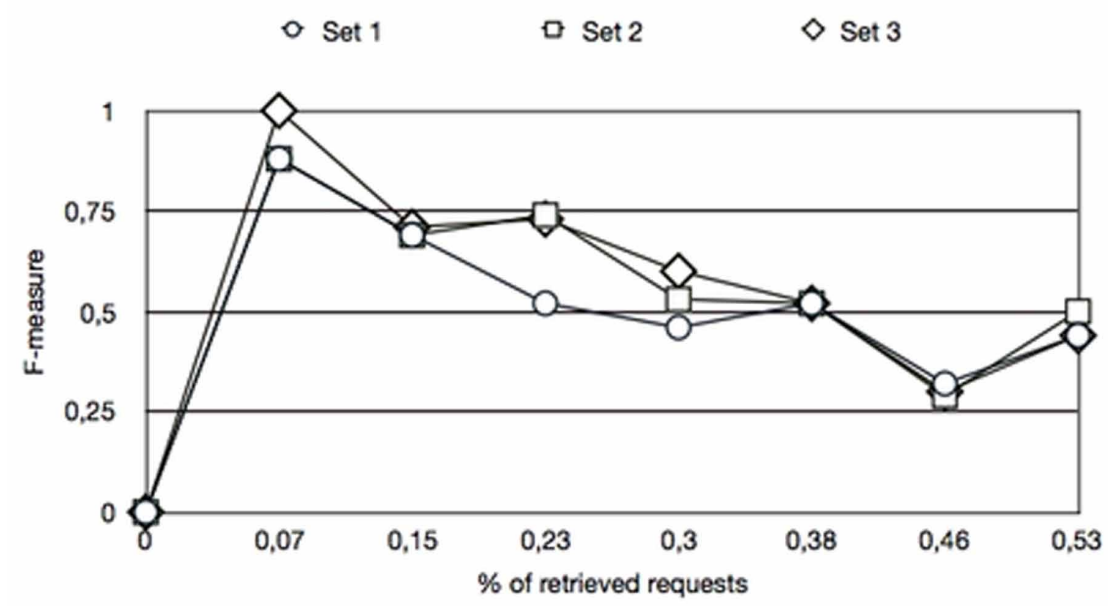

\section{CONCLUSION}

In this paper we presented a relevant knowledge extraction approach based on the automatic construction of temporary fuzzy ontology for the identification and interpretation of user requests. The fuzzy ontology allowed us to automatically construct equivalent system requests and estimate the relevance of these requests. In addition, we can show that it also allows our model to refine the initial user request by presenting the user with a list of equivalent system requests by exploiting the semantic relations between the different objects and user procedures of the ontology. The implementation of the different algorithms allowed us to test the performance of the model and to measure its accuracy and its recall coefficient. We have noticed that our approach has significant precision when the number of proposed requests is low and an acceptable precision when the number of retrieved requests is quite high. We have also noticed that the recall rate of our model is quite high especially after enrichment of the KB. The uses of a fuzzy ontology constructed and enriched automatically by the interaction with the users, make us to easily identify the object and procedure targeted by the user via the semantic links between the system object and user object or between system procedures and user procedures pairs. This strategy represents the originality of our work compared to the few existing works.

As prospects of our work, we first plan to renew different tests using a standard KB to verify and confirm the previous results. We have experimented our approach as a whole to study its effectiveness and verify its performance, and in a short-term job, we plan to conduct a separate test of this ontology to confirm its highlight with regards to the other techniques. The model, we proposed, in its current version can solve user requests with a specific format, a first improvement of the model would be to handle more complex requests by exploiting the user areas and the fuzzy values of the system attributes. A second improvement would be to incorporate a learning process that enriches the user areas of objects and procedures while preserving the consistency and stability of the KB.

\section{ACKNOWLEDGMENT}

Thank you to the anonymous reviewers for their helpful comments and valuable suggestions which have contributed to the final preparation of the paper. 


\section{REFERENCES}

Bouchon, B., Omri, M. N., \& Tijus, C. A. (1994). A Fuzzy Sets Based System for User's Assistance: How SIFADE Diagnoses User's Goals. In Proceedings of the Second Wold Congress on Expert Systems (Vol. 21, pp. 493-498). Academic Press.

Chebil, W., Soualmia, L., Omri, M. N., \& Darmoni, S. J. (2017). Biomedical Documents Indexing with Bayesian Networks and Terminologies. In Proceedings of the 12th International Conference on Intelligent Systems and Knowledge Engineering (Vol. 12, pp. 1-6). IEEE. doi:10.1109/ISKE.2017.8258745

Chebil, W., Soualmia, L. F., \& Omri, M. N. (2018). Possibilistic Information Retrieval Model Based on a MultiTerminology. In Proceedings of the 14th International Conference on Advanced Data Mining and Applications (ADMA) (Vol. 14. pp 66-79). Academic Press. doi:10.1007/978-3-030-05090-0_6

Chebil, W., Soualmia, L. F., Omri, M. N., \& Darmoni, S. J. (2014). Extraction possibiliste de concepts MeSH a partir de documents biomedicaux. Revue d Intelligence Artificielle., 28(6), 729-752. doi:10.3166/ria.28.729-752

Chebil, W., Soualmia, L. F., Omri, M. N., \& Darmoni, S. J. (2016). Indexing biomedical documents with a possibilistic network. Journal of the Association for Information Science and Technology, 67(4), 928-941. doi:10.1002/asi.23435

Cong-Vinh, P. (2009). Categorical Approaches to Models and behaviors of Autonomic Agent Systems. International Journal of Cognitive Informatics and Natural Intelligence, 3(1), 1-33. doi:10.4018/jcini.2009010102

Fkih, F., \& Omri, M. N. (2016). IRAFCA: An O(n) Information Retrieval Algorithm based on Formal Concept Analysis. Knowledge and Information Systems, 48(2), 465-491. doi:10.1007/s10115-015-0876-X

Mendel, J. M., Hagras, H., Tan, W. W., Melek, W. W., \& Ying, H. (2014). Introduction to Type-2 Fuzzy Logic Control: Theory and Applications. Hoboken, NJ: John Wiley \& Sons. doi:10.1002/9781118886540

Naouar, F., Hlaoua, L., \& Omri, M. N. (2013). Relevance Feedback for Collaborative Retrieval Based on Semantic Annotations. In Proceedings of the International Conference on Information and Knowledge Engineering (Vol. 13, pp. 54-60). Academic Press.

Naouar, F., Hlaoua, L., \& Omri, M. N. (2013). Relevance Feedback in Collaborative Information Retrieval based on Validated Annotation. In Proceedings of the International Conference on Reasoning and Optimization in Information Systems (Vol. 1). Academic Press.

Omri, M. N. (2004). Possibilistic Pertinence Feedback and Semantic Networks for Goal's Extraction. Asian Journal of Information Technology, 3(4), 258-265.

Omri, M. N. (2004). Pertinent knowledge extraction from a semantic network: Application of Fuzzy sets theory. International Journal on Artificial Intelligence Tools, 13(3), 705-719.

Omri, M. N. (2004). Relevance feedback for goal's extraction from fuzzy semantic networks. Asian Journal of Information Technology, 3(6), 434-440.

Omri, M. N. (2004). Possibilistic pertinence feedback and semantic networks for goal extraction, Asian journal of information. Technology, 3(4), 258-265.

Omri, M. N. (2012). Effects of Terms Recognition Mistakes on Requests Processing for Interactive Information Retrieval. International Journal of Information Retrieval Research, 2(3), 19-35. doi:10.4018/ijirr.2012070102

Omri, M. N., \& Chouigui, N. (2001). Measure of similarity between fuzzy concepts for identification of fuzzy user requests in fuzzy semantic networks. International Journal of Uncertainty, Fuzziness and Knowledge-based Systems, 9(6), 743-748. doi:10.1142/S0218488501001198

Parry, D. (2002). A fuzzy ontology for medical retrieval. In Proceedings of the ACSW Frontiers '04 second workshop on Australasian information security, Data Mining and Web Intelligence, and Software Internationalisation (vol. 32, pp. 121-126). Academic Press.

Selvachandran, G., Pal, M., Tahani, A., Alhawari, A., \& Salleh, A. (2018). Interval-Valued Complex Fuzzy Sets and its Application to the Malaysian Economy. International Journal of Fuzzy System Applications, 7(1), 22-31. doi:10.4018/IJFSA.2018010102 
Shanshan, M., \& Yingdong, H. (2018). Generalized scaled prioritized intuitionistic fuzzy geometric interaction aggregation operators and their applications to the selection of cold chain logistics enterprises. International Journal of Fuzzy System Applications, 7(2), 1-21.

Sooraj, T. R., \& Tripathy, B. K. (2018). An Interval Valued Fuzzy Soft Set Based Optimization Algorithm for High Yielding Seed Selection. International Journal of Fuzzy System Applications, 7(2), 44-61. doi:10.4018/ IJFSA.2018040102

Suganuma, T., Sugawara, K., Kinoshita, T., Hattori, F., \& Shiratori, N. (2009). Concept of symbiotic computing and its agent-based application to a ubiquitous care-support service. International Journal of Cognitive Informatics and Natural Intelligence, 3(1), 34-56.

Sujit, D., Debashish, M., Samarjit, K., \& Tandra, P. (2018). A Brief Review and Future Outline on Decision Making Using Fuzzy Soft Set. International Journal of Fuzzy System Applications, 7(2), 1-43. doi:10.4018/ IJFSA.2018040101

Uchiya, T., Maemura, T., Hara, H., Sugawara, K., \& Kinoshita, T. (2009). Interactive Design Method of Agent System for Symbiotic computing. International Journal of Cognitive Informatics and Natural Intelligence, 3(1), 57-74. doi:10.4018/jcini.2009010104

Widyantoro, D., \& Yen, J. (2001). A fuzzy Ontology-based Abstract Search Engine and its User Studies. Texas University, Pennsylvania State University. doi:10.1109/FUZZ.2001.1008895

Mohamed Nazih Omri received a PhD degree in Computer Science from the University of Jussieu in 1994, Paris, France. He is an Associate Professor in Computer Science at the Faculty of Sciences of Monastir, in Monastir University, Tunisia. He is the Director of MARS (Modeling of Automated Reasonin Systems) Research Unit. He is also the General Secretary of TCS (Tunisian Computing society). His group conducts research on data mining, text mining, web mining, and information retrieval, indexing methods, and semantic web. 